\title{
Effective removal of field-emitting sites from metallic surfaces by dry ice cleaning
}

\author{
Arti Dangwal| $\left.\right|^{\text {a) }}$ and Günter Müller \\ FB C Physik, Bergische Universität Wuppertal, D-42097 Wuppertal, Germany \\ Detlef Reschke, Klaus Floettmann, and Xenia Singer \\ DESY, Notkestrasse 85, D-22603 Hamburg, Germany
}

(Received 27 February 2007; accepted 6 July 2007; published online 21 August 2007)

\begin{abstract}
Systematic results of the field emission properties of polycrystalline copper and niobium and single-crystal $\mathrm{Nb}$ are reported. Dry ice cleaning (DIC) is found to suppress enhanced field emission from metallic surfaces. The cleaning effect on the emitting sites was investigated by means of field emission scanning microscopy up to fields of $250 \mathrm{MV} / \mathrm{m}$ and high-resolution scanning electron microscopy with energy dispersive x-ray analysis. The number density of emitters at given fields was drastically reduced by dry ice cleaning. Current-voltage measurements and derived Fowler-Nordheim parameters are partially discussed with respect to the morphology and impurity content of localized emitters. No emission from grain boundaries on large-grain $\mathrm{Nb}$ samples was observed. The microscopy results prove the effective removal of field-emitting particulates down to $400 \mathrm{~nm}$ as well as the partial smoothing of surface protrusions by DIC. (C) 2007 American Institute of Physics. [DOI: 10.1063/1.2772505]
\end{abstract}

\section{INTRODUCTION}

Development of effective cleaning techniques for the removal of small particulates from metal surfaces is a primary need in high-voltage vacuum devices. ${ }^{1}$ Micron size surface irregularities can seriously affect the performance of high field accelerator cavities ${ }^{2}$ by creating field emission (FE) sites, ${ }^{3}$ from where electrons are emitted at much lower electric fields than predicted by Fowler-Nordheim (FN) theory. ${ }^{4}$ This enhanced FE is mainly caused by particulates ${ }^{5}$ and surface protrusions ${ }^{6}$ due to local field enhancement. Such emitters originate as residues of chemical surface preparation and cleaning techniques, or result from insufficient cleanroom conditions and mishandling of the surface. ${ }^{7-10}$

The removal of particulate emitters is rather difficult because these usually stick to the solid surface due to adhesion. The interaction between particulates and substrate includes van der Waals, electrostatic, and capillary forces. ${ }^{11}$ All these adhesion forces $F$ are found to be proportional to the particulate diameter $d$. Accordingly, a particulate of mass $m \sim d^{3}$ will require an acceleration $a=F / m \sim 1 / d^{2}$, which becomes quadratically larger with decreasing size. Additionally, the number density of ambient particulates usually increases with decreasing size. Therefore, some small particulates usually remain on the surface after conventional cleaning techniques used in accelerator technology such as high-pressure rinsing (HPR) with ultrapure water. ${ }^{12}$

Dry ice cleaning (DIC) is a powerful technique which uses a high-pressure jet of pure carbon dioxide snow to loosen and remove different types of particulate contaminations from the surface by its unique combination of thermal, mechanical, and chemical effects. ${ }^{13-16}$ The rapid cooling at

\footnotetext{
${ }^{a)}$ Corresponding author; FAX: + 4920243928 11; Electronic mail: arti@physik.uni-wuppertal.de
}

the point of hitting brittles the particulates, thus weakening the adhesion for their efficient removal by the impact of snow particles. Unlike HPR, this technique significantly removes hydrocarbon contaminations from the cleaned surfaces ${ }^{13}$ since carbon dioxide is a good solvent for nonpolar chemicals. The possibility of a dry in situ cleaning of the cavities, and already proven faster pump-down rates with DIC,${ }^{17}$ are surely advantageous for accelerator technology.

Nowadays copper and niobium sheet metals are used for the fabrication of cavities for accelerators. Dark currents caused by enhanced FE limit the accelerating field gradients in normal conducting $\mathrm{rf}$ guns ${ }^{18}$ and superconducting $\mathrm{Nb}$ cavities. ${ }^{19}$ Therefore, systematic investigations of the dc field emission properties of relevant metallic samples has proven to be helpful for the optimization of surface preparation techniques. In order to understand the origin of the emitters, field emission scanning microscopy (FESM) ${ }^{6,20}$ combined with high-resolution secondary electron microscopy (SEM) and energy dispersive $\mathrm{x}$-ray analysis (EDX), is required. ${ }^{10}$

Here, we report on the effect of DIC on $\mathrm{Cu}$ and $\mathrm{Nb}$ surfaces by (i) comparing the number density of FE sites on particular sample areas scanned in FESM before and after DIC; (ii) determining the FE properties of localized emitters by current versus voltage $(I-V)$ measurements and FN analysis; and (iii) investigating their morphology and composition by SEM and EDX. This comprehensive study will prove that DIC reduces FE efficiently by removal of micron and submicron particulates as well as by smoothing of surface protrusions.

\section{EXPERIMENTAL TECHNIQUES}

We have tested four copper ( $7 \mathrm{~mm}$ squares) and six niobium ( $28 \mathrm{~mm}$ diameter) samples, of which two were always fabricated with the same techniques for reproducibility 


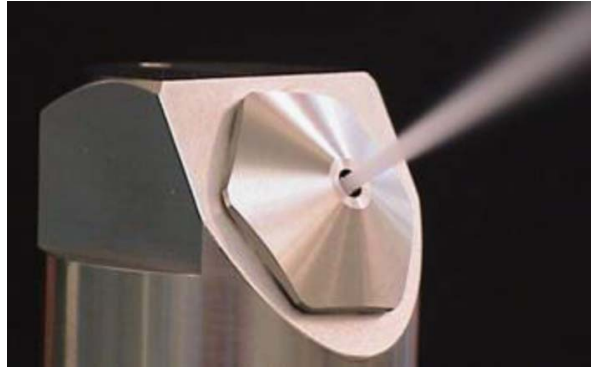

FIG. 1. (Color online) High-pressure dry-ice jet emerging from DIC nozzle.

checks. Two polycrystalline $\mathrm{Cu}$ samples were cut from a regularly turned cavity cell and the other two from a singlepoint diamond-turned cavity cell. Two polycrystalline $\mathrm{Nb}$ samples (PolyNb) were machined from high-purity niobium with a residual resistance ratio RRR of about 300 and electropolished up to $140 \mu \mathrm{m}$ using $\mathrm{H}_{2} \mathrm{SO}_{4}$ : $\mathrm{HF}$ in 9:1 volume ratio, resulting in a surface roughness of less than $0.5 \mu \mathrm{m}$. Two large-grain $(\sim \mathrm{cm})$ and two single-crystal $\mathrm{Nb}$ samples $(\mathrm{CryNb})$ of $\mathrm{RRR} \sim 340$ were chemically etched by buffered chemical polishing ( $\mathrm{HF}: \mathrm{HNO}_{3}: \mathrm{H}_{3} \mathrm{PO}_{4}$ in volume ratio $1: 1: 2)$ to remove the damage layer up to $30 \mu \mathrm{m}$, resulting in a mirrorlike surface with surface roughness of less than $0.1 \mu \mathrm{m}$. Finally, all samples were extensively rinsed with ultrapure water and HPR in the case of Nb. All the samples have been especially marked during fabrication to adjust the sample position in different experimental setups. For the sample transfer between the laboratories, an approved protection cap system ${ }^{21}$ was used and opened under cleanroom or high-vacuum conditions only.

A modified DIC of the samples was performed in a class 10 cleanroom. Detailed description of the DIC experimental setup and the cleaning procedure has been described elsewhere. ${ }^{22-24}$ Carbon dioxide of purity grade $4.5\left(\mathrm{~N}_{2}+\mathrm{O}_{2}\right.$ $<45$ vol ppm., $\mathrm{H}_{2} \mathrm{O}<5$ vol ppm., $\mathrm{C}_{n} \mathrm{H}_{m}<1$ vol ppm.) and pure nitrogen (both particle filtered to $<0.05 \mu \mathrm{m}$ ) are supplied up to pressures of 50 and 12 bar, respectively. Figure 1 shows the high-pressure DIC jet coming out of a dedicated nozzle system developed at Fraunhofer Institut Produktionstechnik und Automatisierung (Stuttgart, Germany) for DESY for the cleaning of cavities. The $\mathrm{CO}_{2}$ snow jet is surrounded by supersonic nitrogen, ${ }^{22}$ which provides additional acceleration and focusing of the jet as well as a partial pre-

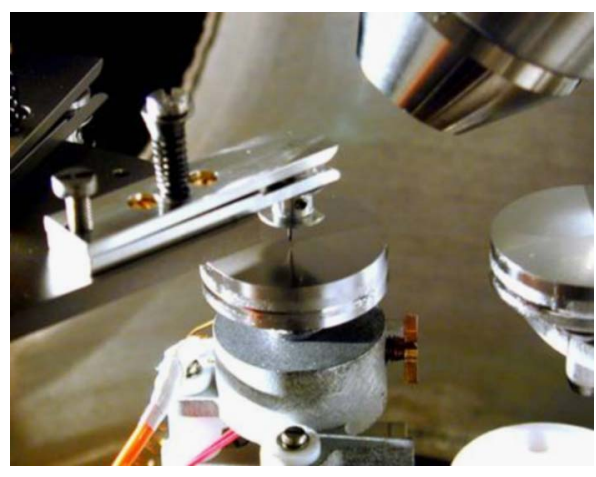

FIG. 2. (Color online) Inner view of FESM, showing a mirrorlike $\mathrm{Nb}$ sample of $28 \mathrm{~mm}$ diameter below a tungsten tip anode $(\sim 1 \mu \mathrm{m}$ apex radius).
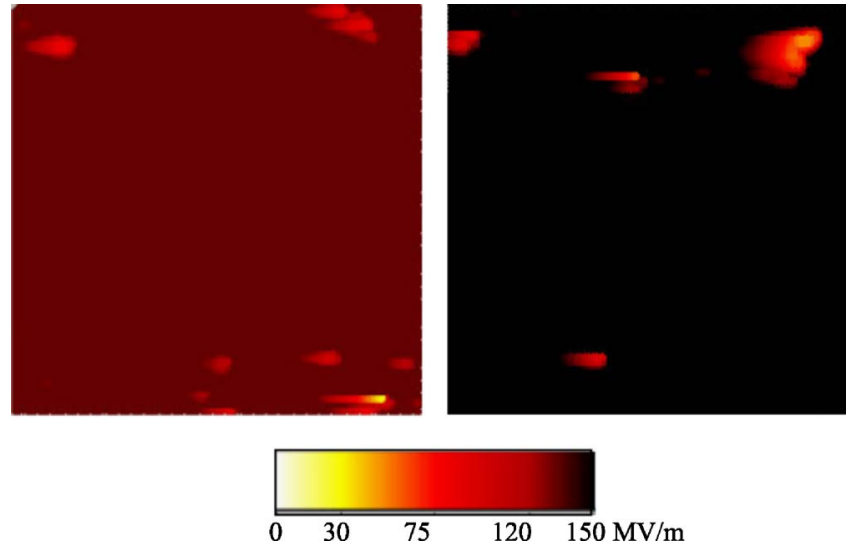

FIG. 3. (Color online) FE maps of $\mathrm{Cu}$, performed with a conical anode $(100 \mu \mathrm{m})$ over the same $(5 \times 5) \mathrm{mm}^{2}$ area before (left) and after (right) DIC at 120 and $150 \mathrm{MV} / \mathrm{m}$, respectively.

vention of moisture condensation at the cleaned surface. The temperature of liquid $\mathrm{CO}_{2}$ is maintained between $-18{ }^{\circ} \mathrm{C}$ and $-10{ }^{\circ} \mathrm{C}$ at the dedicated cooler/purifier unit, ${ }^{16}$ which is connected to the nozzle by a pipe of $3 \mathrm{~m}$ length. This configuration keeps the fraction of snow in the jet on the order of $45 \%{ }^{22}$ An effective gas extraction system and a controlled heating system avoid recontamination and humidity condensation at the sample surface, respectively. The flat samples were cleaned by moving the DIC nozzle linearly back and forth with a constant speed. The cleaning duration of the large samples (28 $\mathrm{mm}$ diameter) was usually about $5 \mathrm{~min}$, which includes the intermediate time for rotating the sample six times manually by $30^{\circ}$.

FESM measurements have been made in two steps: (i) scans over a selected sample area with a fixed anode at a maximum field given by a high applied voltage $V$ regulated for a constant current $I(2 \mathrm{nA})$ within a few milliseconds, resulting in maps of the found emitters at correspondingly reduced field level; and (ii) local characterization of the interesting emitters observed in the scans. The scans were performed at field levels varying as 60, 90, 120, 150, 200, and $250 \mathrm{MV} / \mathrm{m}$ for different samples by using a $5 \mathrm{kV}$ power supply and adjusting the gaps between sample and anode down to $20 \mu \mathrm{m}$. For the scans conical anodes of diameter 300 or $100 \mu \mathrm{m}$ were used depending on the area and required resolution. Local measurements were mostly performed with tip anodes of diameters 30,10 , or smaller down to $1 \mu \mathrm{m}$ to resolve small emitting sites. Figure 2 shows a tip anode of apex radius $1 \mu \mathrm{m}$ placed just above an emitting site on a large-grain $\mathrm{Nb}$ sample. The mirrorlike sample surface is helpful to control the gap within an accuracy of $\pm 5 \mu \mathrm{m}$ by observing the anode image with a long-distance microscope and charge-coupled device (CCD) video camera. The real electrode distance $z$ is derived prior to any local $I$ $-V$ curve of an emitter by extrapolation of the measured $V(z)$ plot. ${ }^{20}$ The local onset field for a current of $2 \mathrm{nA}, E_{\text {on }}$ $=V /(z \alpha)$, has been corrected for the calculated tip shape factor $\alpha$.

The relocalization of the emitters found with FESM in an ex situ SEM has been ensured within an accuracy of $\pm 500 \mu \mathrm{m}$ by using the marks on the samples. The high- 


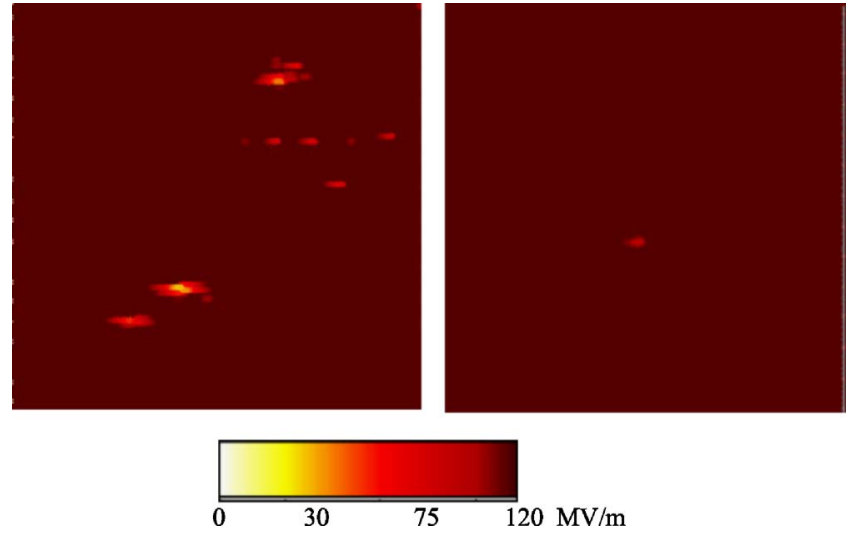

FIG. 4. (Color online) FE maps of polycrystalline $\mathrm{Nb}$, performed with a conical anode $(100 \mu \mathrm{m})$ over the same $(7.5 \times 7.5) \mathrm{mm}^{2}$ area before (left) and after (right) DIC at $120 \mathrm{MV} / \mathrm{m}$.

resolution SEM images and EDX measurements (for elements with $Z>10$ ) were made by means of a Philips XL-30 with $\mathrm{LaB}_{6}$ cathode.

\section{RESULTS AND DISCUSSION}

\section{A. Statistical reduction of FE by DIC}

FE scans of two $\mathrm{Cu}$ samples (one of each type) performed with $100 \mu \mathrm{m}$ conical anode over the area of (5 $\times 5) \mathrm{mm}^{2}$ showed no emission at $60 \mathrm{MV} / \mathrm{m}$ before DIC. For the diamond-turned sample, 10 emitters have been found at $120 \mathrm{MV} / \mathrm{m}$ as shown in Fig. 3(a), which reduced to 1 after DIC. This improved surface gives 6 emitters at $150 \mathrm{MV} / \mathrm{m}$ [Fig. 3(b)]. The regularly turned $\mathrm{Cu}$ sample gave 8 emitters at $90 \mathrm{MV} / \mathrm{m}$, which improved after DIC to 0 (3) emitters at 90 (120) MV/m, i.e., very comparable results. Therefore, the remaining $\mathrm{Cu}$ samples were directly dry ice cleaned. All four DIC Cu samples showed no FE up to $90 \mathrm{MV} / \mathrm{m}$, and the best one up to $120 \mathrm{MV} / \mathrm{m}$. No significant difference was found between the regularly and diamond-turned $\mathrm{Cu}$ samples.

The PolyNb samples were first scanned with $300 \mu \mathrm{m}$ anode over the area of $(12 \times 12) \mathrm{mm}^{2}$ at low fields $(40,60,90$ $\mathrm{MV} / \mathrm{m})$ and then with $100 \mu \mathrm{m}$ anode over $(7.5 \times 7.5) \mathrm{mm}^{2}$ area at $120 \mathrm{MV} / \mathrm{m}$. Both samples showed before DIC first emission of $(1,2)$ emitters at $60 \mathrm{MV} / \mathrm{m}$, which disappeared
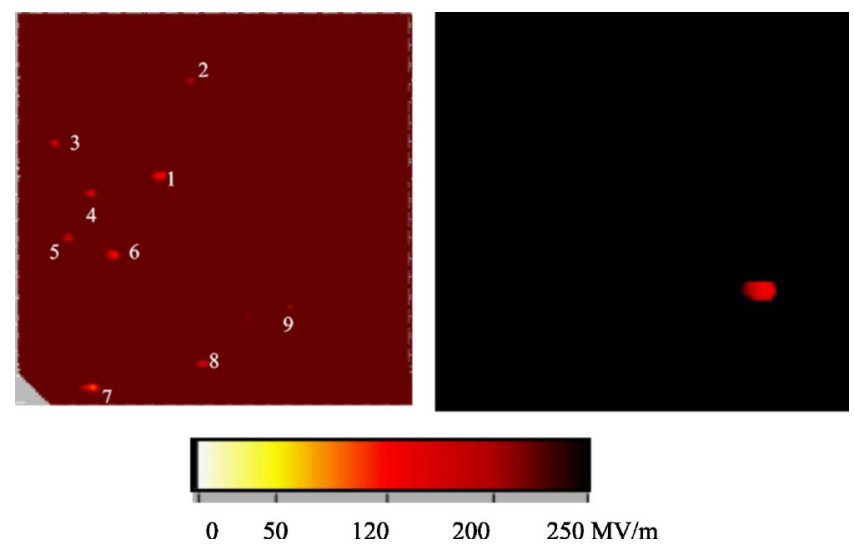

FIG. 5. (Color online) FE maps of single-crystalline Nb, performed with a conical anode $(100 \mu \mathrm{m})$ over the same $(5 \times 5) \mathrm{mm}^{2}$ area before (left) and after (right) DIC at 200 and $250 \mathrm{MV} / \mathrm{m}$, respectively.

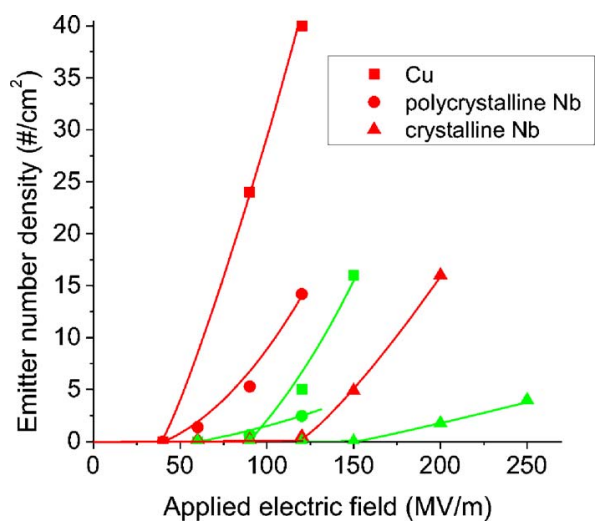

FIG. 6. (Color online) Emitter number density vs applied electric field for showing the improvement by DIC for the different materials. (Dark symbols (red online): before DIC, and grey symbols (green online): after DIC).

after DIC and improved to $(2,0)$ emitters at $90 \mathrm{MV} / \mathrm{m}$ and $(2,3)$ at $120 \mathrm{MV} / \mathrm{m}$. The great improvement after DIC for one of the samples is shown in Fig. 4. Only one emitter survived but has been weakened by DIC, as seen from the field scales of the maps. For a more detailed interpretation, strong emitters were investigated locally in FESM and SEM and will be discussed in Secs. III B and III C.

FE maps of CryNb samples have shown very good results even before DIC: for large-grain $\mathrm{Nb}(0,2)$ at 120 $\mathrm{MV} / \mathrm{m},(5,3)$ at $150 \mathrm{MV} / \mathrm{m}$, and $(10,12)$ at $200 \mathrm{MV} / \mathrm{m}$, and for single-crystal $\mathrm{Nb}$ no emission up to $120 \mathrm{MV} / \mathrm{m},(2,1)$ emitters at $150 \mathrm{MV} / \mathrm{m}$, and $(5,9)$ at $200 \mathrm{MV} / \mathrm{m}$. It is most interesting that DIC further improves such high-quality surfaces. Three samples, one large-grain $\mathrm{Nb}$ and two single crystal, were dry ice cleaned and showed no FE up to 150 $\mathrm{MV} / \mathrm{m}$, but $(2,0,1)$ emitters at 200 and $250 \mathrm{MV} / \mathrm{m}$. FE maps of one sample at the maximum fields before and after DIC are given in Fig. 5. The main observations are (i) removal of all old emitters after DIC, showing the effectiveness of this cleaning technique; and (ii) existence of one new emitter at $250 \mathrm{MV} / \mathrm{m}$ (which occurred already at $200 \mathrm{MV} / \mathrm{m}$ ).

A statistical overview of the number density of emitters $N$ for the varying electric field $E$ is presented in Fig. 6. In order to reduce the statistical error and to simplify the $N(E)$ plot, we summed up all the results for a particular kind of sample, i.e., the corresponding areas and number of emitters were added at the scanned field levels. Thus, only six lines for the three different kinds of samples before and after DIC summarize all the scan results. From the statistical plot, the beneficial effect of DIC on these metallic surfaces is obvious: (i) shift of $N(E)$ curves to right, i.e., onset of FE observed at higher fields; and (ii) $N(E)$ curves with much reduced slope, i.e., huge reduction of the number density of field emitters at a given field level. Within statistical errors, for $\mathrm{Cu}$ and PolyNb no FE was observed up to $60 \mathrm{MV} / \mathrm{m}$ before and $90 \mathrm{MV} / \mathrm{m}$ after DIC, and for CryNb up to 150 and $200 \mathrm{MV} / \mathrm{m}$, respectively. It is remarkable that one of the DIC single-crystal samples did not show any FE up to $250 \mathrm{MV} / \mathrm{m}$.

\section{B. FN analysis and stability of emitters}

The majority of the localized emitters on all scanned samples were analyzed with FESM. The current-voltage 


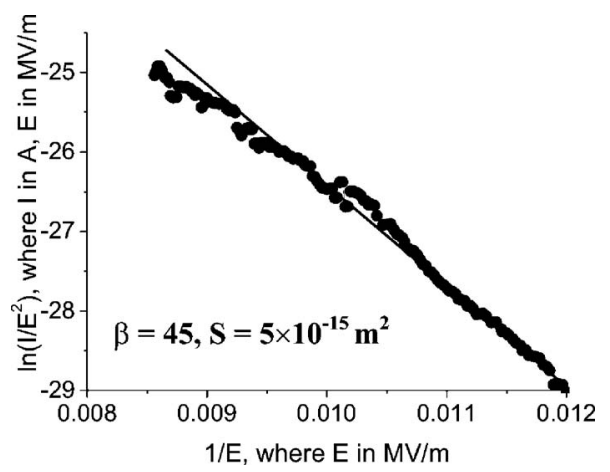

FIG. 7. $I-V$ curve for a typical emitter on dry ice cleaned $\mathrm{Cu}$ sample, with $\beta, S$ values derived for $\Phi=4.4 \mathrm{eV}$ from its $\mathrm{FN}$ fit (solid line).

characteristics of the emitting sites were found to obey the modified FN law. ${ }^{3}$ Accordingly, the linear fitting of $\ln \left(I / E^{2}\right)$ vs $1 / E$ plot gives the field enhancement factor $\beta$ for a given work function $\Phi$ from its slope and the effective emission area $S$ from its intercept. Figure 7 shows an $I-V$ curve for a typical emitter on DIC $\mathrm{Cu}$ surface, resulting in a characteristic $\beta$ value of 45 and $S$ value of $0.005 \mu \mathrm{m}^{2}$. These values seem to be reasonable for a particulate or protrusion of size $h(\sim \mu \mathrm{m})$ and sharp edge radius $r(\sim 10 \mathrm{~nm})$, since $\beta=h / r$ and $S=r^{2}$.

The impact of DIC on individual emitters can be seen by the changes in their respective $I-V$ curves before and after cleaning, as shown for PolyNb in Fig. 8. The large shift to the left means a drastic increase of the $E_{\text {on }}$ values, both for a

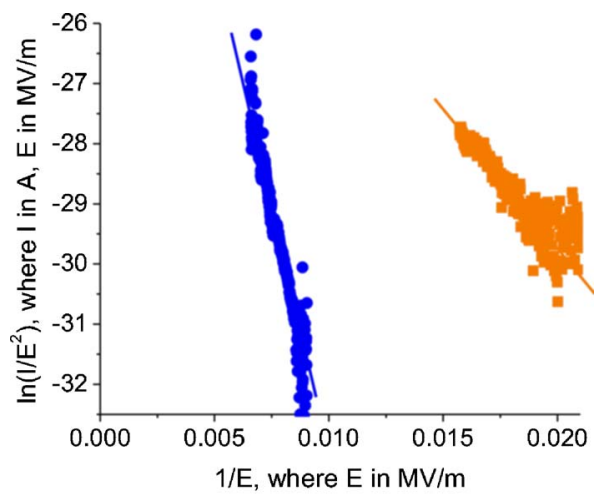

(a)

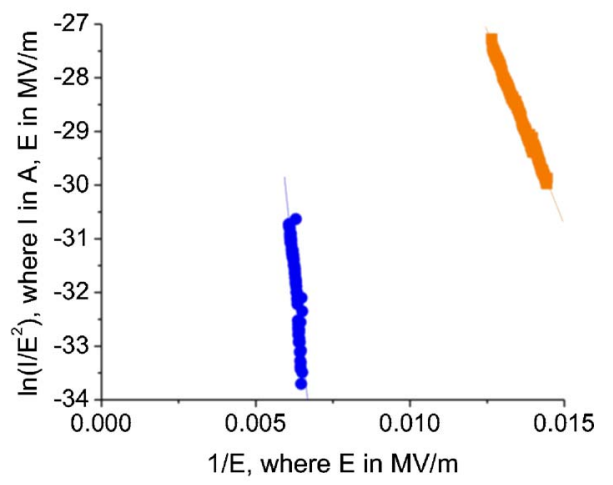

(b)

FIG. 8. (Color online) FN plots for a typical particulate (a) and protrusion (b) on electropolished $\mathrm{Nb}$ sample before $(\boldsymbol{\bullet}$, light/orange) and after $(\boldsymbol{\bullet}$, dark/blue) DIC.

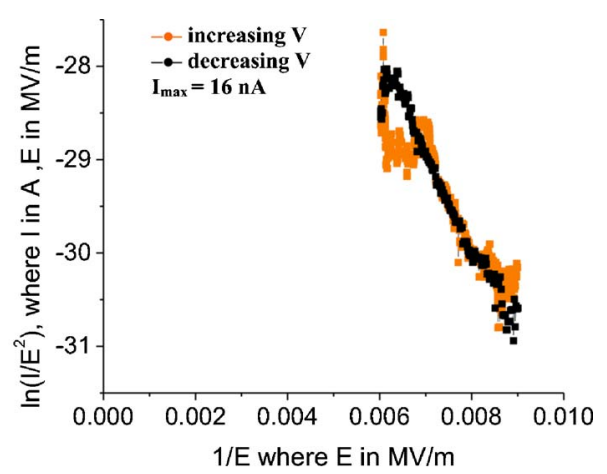

(a)

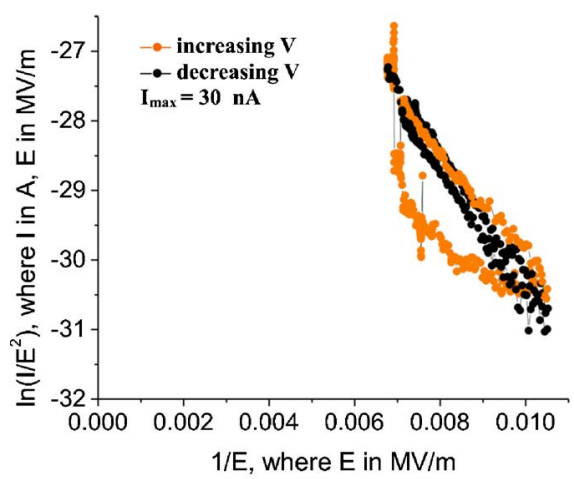

(b)

FIG. 9. (Color online) FN plots for a typical particulate (a) and scratch (b) on $\mathrm{BCP}$-treated single-crystal $\mathrm{Nb}$ sample showing current processing effects.

scratch and a particulate. The changes observed in the derived $\beta$ and $S$ values are as follows: $\beta$ decreased from 51 to 38 for the particulate and from 148 to 31 for the scratch, while $S$ increased from $1 \times 10^{-15}$ to $2 \times 10^{-13} \mathrm{~m}^{2}$ for the former and from $7 \times 10^{-20}$ to $3 \times 10^{-16} \mathrm{~m}^{2}$ for the latter. Decreased $\beta$ and increased $S$ values can be understood by the smoothened edges of the emitters after DIC. It is remarkable that the particulate shows more current fluctuation than the scratch, as expected from their relative mechanical stability.

Often such current fluctuations lead to preliminary deviations from $\mathrm{FN}$ behavior, which can usually be minimized by high current processing. The $I-V$ curves for a typical particulate on CryNb [Fig. 9(a)] show: (i) FN behavior with increasing field, which seems to be saturated on reaching currents of few nA, but is finally followed by a current jump; (ii) the curve retraces more stably for decreasing field values. While the stabilized FN fit values $\left(\beta=48, S=9 \times 10^{-19} \mathrm{~m}^{2}\right)$ suggest an unchanged geometry of the emitter, the preliminary saturation hints for adsorbate effects. ${ }^{25}$ The $I-V$ curves for a scratch are given in Fig. 9(b), where the emitter has been irreversibly activated at a current of $30 \mathrm{nA}$ and then attains a stable FN behavior $\left(\beta=61, S=3 \times 10^{-19} \mathrm{~m}^{2}\right)$ in consecutive cycles of increasing and decreasing voltages. In general comparison, the $I-V$ curves of particulates showed more instabilities than surface protrusions.

In total, 54 emitters from all samples have been measured locally for their $I-V$ curves. Representative results for selected emitters are given in Table I, where their characteristic $E_{\mathrm{on}}, \beta$, and $S$ values before and partially after DIC are 
given. The range of $E_{\text {on }}$ in $\mathrm{MV} / \mathrm{m}$ before DIC is $49-84$ for $\mathrm{Cu}$ and PolyNb, and has been improved by DIC to $63-110$ for $\mathrm{Cu}$ and PolyNb. Significantly higher values, i.e., a range of 88-167 before and 116-186 after DIC, were found for $\mathrm{CryNb}$ and underline the benefit of mirrorlike surfaces for reduced FE. Most of the $\beta$ values, i.e., $25-56$ for $\mathrm{Cu}, 18-148$ for PolyNb, and 17-76 for CryNb, are inversely correlated with the $E_{\text {on }}$ values. The derived $S$ parameters cover rather large ranges, i.e., 30 to $3 \times 10^{-6} \mu \mathrm{m}^{2}$ for $\mathrm{Cu}$ and 0.2 to 3 $\times 10^{-7} \mu \mathrm{m}^{2}$ for all $\mathrm{Nb}$ samples, most of which are reasonable with respect to the $\beta$ values. The extreme values, however, cannot be understood within the modified FN theory.

In order to check if grain boundaries cause enhanced FE, the large-grain CryNb samples were scanned $\left(1 \mathrm{~mm}^{2}\right)$ with $30 \mu \mathrm{m}$ resolution over the grain boundary intersections. No emission was observed up to the highest scanned field levels. Despite the fact that grain boundaries on $\mathrm{Nb}$ surfaces can be easily contaminated by segregated impurities, this result suggests that the grain boundary effects play only a minor role for enhanced $\mathrm{FE}$ of clean $\mathrm{Nb}$ samples.

\section{Morphology and composition of emitters}

In order to understand the nature and origin of enhanced FE from metallic surfaces, a total of 31 emitters were relocalized and examined with high-resolution SEM and EDX. Figure 10 shows SEM images of various emitters on DIC Cu samples partially with the corresponding EDX spectra. The submicron particulate $(\beta=38)$ with sulfur contaminant in Fig. 10(a) seems to be embedded in the surface, and its dark contrast suggests a conducting nature. Similar $S$ contaminations up to $5 \mu \mathrm{m}$ size were found at the emitting sites ( $\beta$ up to 41). Si impurities inside a $10 \mu \mathrm{m}$ large fissure [Fig. 10(b), $\beta=32]$ also survived after DIC. Thus, the foreign elements embedded in the surface or trapped inside the grooves could
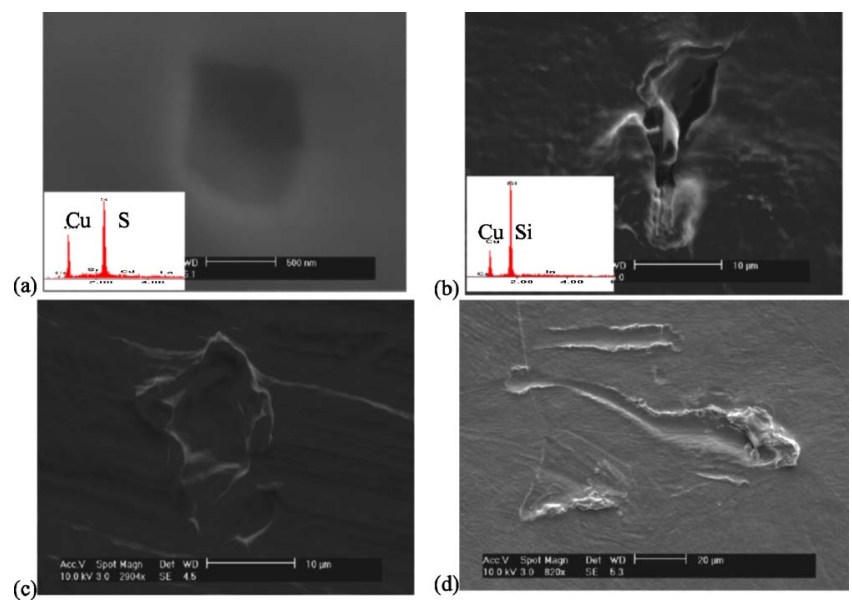

FIG. 10. (Color online) SEM images with EDX spectra (insets) of FE sites on DIC Cu: (a) sulfur contaminant of submicron size embedded in the surface; (b) silicon impurities inside a fissure; (c) surface irregularity; and (d) large scratch.

not be removed completely by DIC. Moreover, the mark of $10 \mu \mathrm{m}$ size [Fig. 10(c), $\beta=56$ ] and the large scratch of about $20 \mu \mathrm{m}$ width [Fig. 10(d), $\beta=41$ ] were identified as emitters with sharp edges and delaminations, thus causing the enhanced FE.

SEM images of some of the emitters found on PolyNb samples are shown in Fig. 11. The particulate of about $50 \mu \mathrm{m}$ length in Fig. 11(a) contains $S, \mathrm{Cl}$, and $\mathrm{K}$ and provides a rather small $\beta$ of 18 . Despite its resistance against HPR, it was completely removed by DIC. Usually all the localized field emitting particulates disappeared after DIC treatment of the samples. Surface irregularities like the mark in Fig. 11(b) reflect mishandling of the surface. In one case, a thin flakelike object of about $20 \mu \mathrm{m}$ size [Fig. 11(c)]was

TABLE I. FESM and SEM/EDX results for selected emitters on ten broad area Cu and Nb cathodes. The FN fit parameters are derived for $\Phi$ of 4.4 eV (Cu) and $4 \mathrm{eV}(\mathrm{Nb})$. $\mathrm{PNb}$ and $\mathrm{CNb}$ corresponds to PolyNb and CryNb samples, respectively.

\begin{tabular}{|c|c|c|c|c|c|c|c|}
\hline \multirow[t]{2}{*}{ Sample_emitter no. } & \multirow{2}{*}{$\mathrm{DIC}$} & \multirow{2}{*}{$E_{\mathrm{on}}(M V / m)$} & \multicolumn{2}{|c|}{ FN fit parameters } & \multirow{2}{*}{$\begin{array}{l}\text { Element detected } \\
\text { by EDX }\end{array}$} & \multirow[t]{2}{*}{ SEM observation } & \multirow[t]{2}{*}{ Related figures } \\
\hline & & & $\beta$ & $S\left(\mu \mathrm{m}^{2}\right)$ & & & \\
\hline Cu1_1 & & 67 & 25 & 30 & n.m. & n.m. & \\
\hline $\mathrm{Cu} 1 \_3$ & & 84 & 41 & $2 \times 10^{-3}$ & n.m. & n.m. & \\
\hline $\mathrm{Cu} 1 \_2$ & $\mathrm{Y}$ & 101 & 38 & $7 \times 10^{-4}$ & $\mathrm{~S}, \mathrm{Cu}$ & $0.6 \mu \mathrm{m}$ embedded particulate & $10(\mathrm{a})$ \\
\hline $\mathrm{Cu} 1 \_4$ & Y & 105 & 32 & $3 \times 10^{-4}$ & $\mathrm{Si}, \mathrm{Cu}$ & Contamination inside fissure & $10(\mathrm{~b})$ \\
\hline $\mathrm{Cu} 2 \_1$ & Y & 103 & 41 & $7 \times 10^{-4}$ & $\mathrm{Cu}$ & Scratch with delamination & $10(d)$ \\
\hline $\mathrm{Cu} 4 \_1$ & $\mathrm{Y}$ & 110 & 56 & $3 \times 10^{-6}$ & $\mathrm{Cu}$ & $10 \mu \mathrm{m}$ mark & $10(\mathrm{c})$ \\
\hline PNb1_2 & & 54 & 51 & $1 \times 10^{-3}$ & $\mathrm{Nb}$ & $20 \mu \mathrm{m}$ particulate & 8(a) and 11(c) \\
\hline $\mathrm{PNb} 1 \_2^{\mathrm{a}}$ & $\mathrm{Y}$ & 63 & 38 & $2 \times 10^{-1}$ & $\mathrm{Nb}$ & Embedded parts & $8(\mathrm{a})$ and $11(\mathrm{~d})$ \\
\hline PNb1_3 & & 49 & 148 & $7 \times 10^{-8}$ & $\mathrm{Nb}$ & Scratch & $8(b)$ \\
\hline $\mathrm{PNb} 1 \_3^{\mathrm{a}}$ & $\mathrm{Y}$ & 103 & 31 & $3 \times 10^{-4}$ & $\mathrm{Nb}$ & Scratch & $8(b)$ and $11(b)$ \\
\hline PNb1_4 & & 77 & 18 & $5 \times 10^{-1}$ & $\mathrm{~S}, \mathrm{Cl}, \mathrm{K}$ & $50 \mu \mathrm{m}$ particulate & $11(\mathrm{a})$ \\
\hline $\mathrm{CNb} 1 \_2$ & & 88 & 71 & $2 \times 10^{-6}$ & $\mathrm{Nb}$ & No feature found & \\
\hline CNb3_1 & & 128 & 48 & $9 \times 10^{-7}$ & $\mathrm{Nb}$ & $1 \mu \mathrm{m}$ particulate & $9(\mathrm{a})$ \\
\hline CNb4_1 & & 127 & 61 & $3 \times 10^{-7}$ & $\mathrm{Si}, \mathrm{Nb}$ & Scratch \& $0.5 \mu \mathrm{m}$ particulate & $9(\mathrm{~b})$ \\
\hline CNb4_6 & & 142 & 59 & $7 \times 10^{-8}$ & $\mathrm{Nb}$ & $1 \mu \mathrm{m}$ particulate & 5 \\
\hline CNb4_5 & & 167 & 75 & $4 \times 10^{-9}$ & $\mathrm{Nb}$ & No feature found & 5 \\
\hline $\mathrm{CNb} 1 \_1$ & $\mathrm{Y}$ & 116 & 18.6 & $3 \times 10^{-2}$ & $\mathrm{Nb}$ & No feature found & \\
\hline CNb1_5 & Y & 123 & 18 & $6 \times 10^{-2}$ & $\mathrm{Nb}$ & No feature found & \\
\hline CNb4_2 & $\mathrm{Y}$ & 186 & 22 & $4 \times 10^{-3}$ & $\mathrm{Nb}$ & No feature found & \\
\hline
\end{tabular}

${ }^{\mathrm{a}}$ Same emitter reidentified after DIC; n.m. means not measured. 


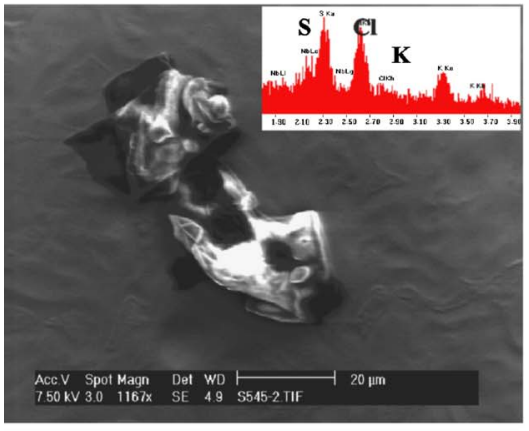

(a)

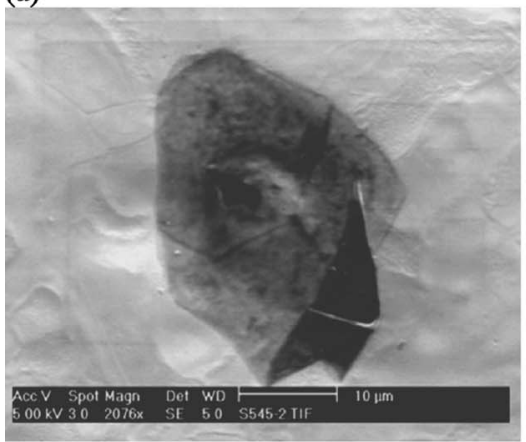

(c)

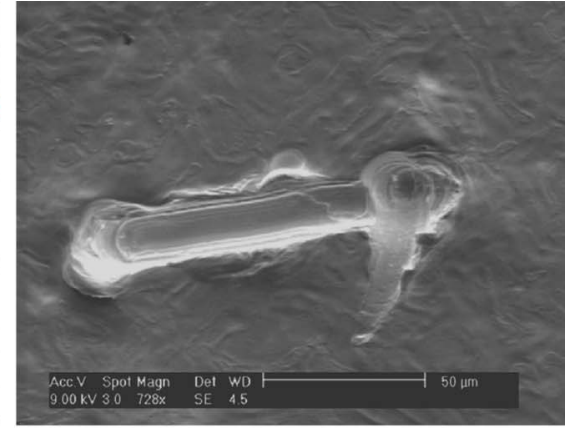

(b)

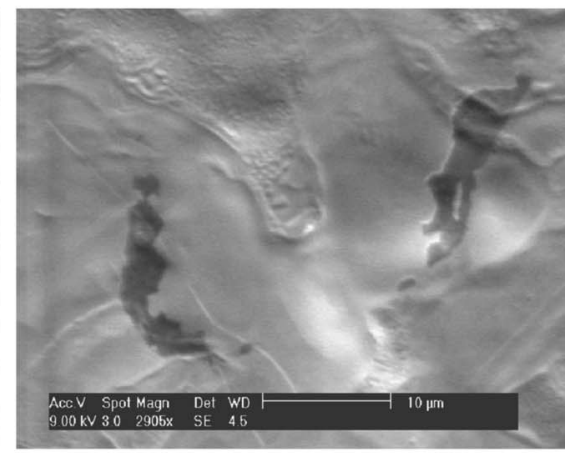

(d)
FIG. 11. (Color online) SEM images of FE sites on polycrystalline $\mathrm{Nb}$ : (a) particulate containing sulfur, chlorine, and potassium (see the inset EDX spectrum) before DIC; (b) large irregularity on the sample surface after DIC; (c) flakelike object before and (d) after DIC, still giving FE. partially destroyed by DIC and only some embedded part of it survived [Fig. 11(d)] and still emitted, as shown in Fig. 8(b). These facts altogether prove the mechanical and thermal effect of DIC on the particulates for the reduction of FE.

On CryNb samples, the particulates localized as emitters before DIC were always removed by DIC. The SEM images given in Figs. 12(a) and 12(b) prove the removal of a particulate as small as $400 \mathrm{~nm}$ in size by DIC from the singlecrystal $\mathrm{Nb}$ surface. The high-resolution SEM images of the scratch head [Figs. 12(c) and 12(d)] show softened contours of some protrusions, probably due to the mechanical impact of high-speed dry-ice particles impinging on the surface. It is remarkable that the $\mathrm{Si}$ contaminant disappeared after DIC. No FE from this site is observed up to $250 \mathrm{MV} / \mathrm{m}$, i.e., there seems to be no features with $\beta$ values above about 10 . These results show the strength of the DIC technique even for the removal of submicron particulates as well as for the partial smoothing of surface protrusions.

From the observed FE properties and SEM/EDX results (Table I), the emitters can be categorized as follows: (i) particulates with or without contaminants; (ii) features embedded in the surface; (iii) surface protrusions with or without
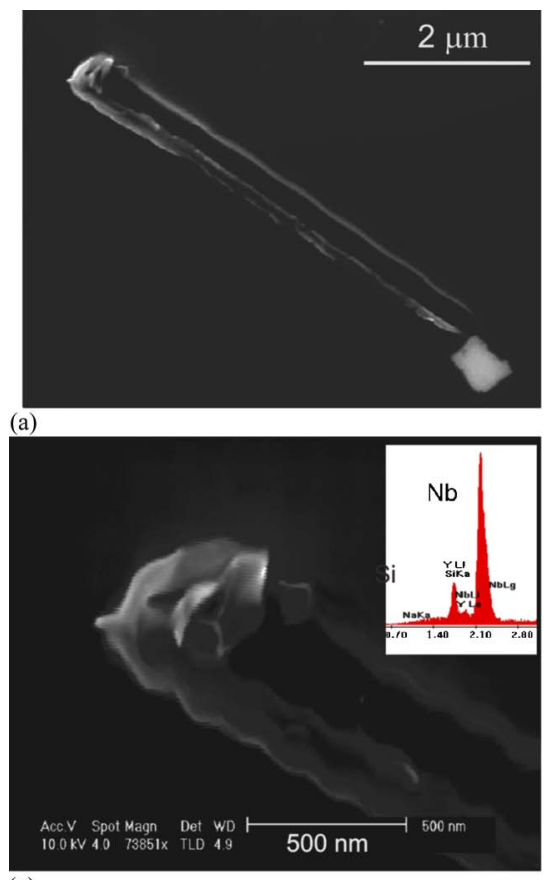

(c)

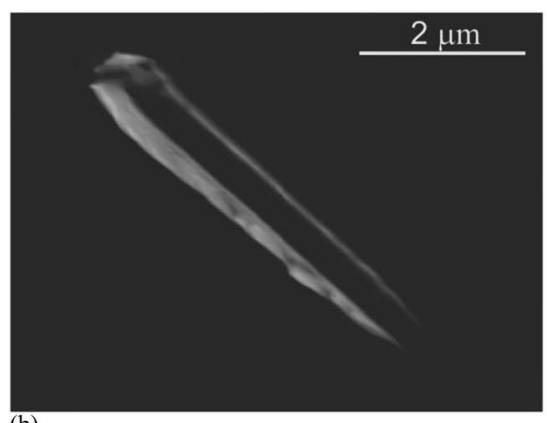

(b)

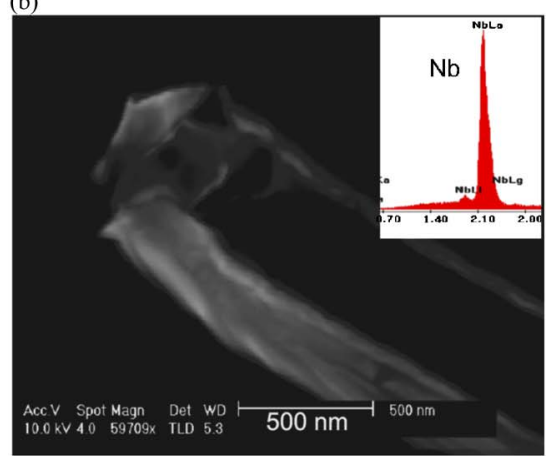

(d)
FIG. 12. (Color online) SEM images of $\mathrm{FE}$ sites on single-crystal $\mathrm{Nb}$ : scratch with a nearby lying submicron particulate of size $\sim 400 \mathrm{~nm}$ before (a) and after (b) DIC, high-resolution picture and EDX spectra of the scratch head before (c) and after (d) DIC. 
contaminants; and (iv) grooves with trapped contaminants. DIC effectively removes emitters of type (i), and weakens emitters of type (iii), while types (ii) and (iv) are less affected. It should be remarked that 19 out of 51 localized emitters did not show any feature in high-resolution ( $<5 \mathrm{~nm})$ SEM. This probably hints at the presence of some embedded impurities with low work function.

\section{CONCLUSIONS}

DIC has been found to be a very efficient technique for the reduction of $\mathrm{FE}$ sites from $\mathrm{Cu}$ and $\mathrm{Nb}$ surfaces. The remaining emitters found on various FE maps statistically appear at much higher electric fields. The best DIC singlecrystal $\mathrm{Nb}$ sample did not provide any FE up to $250 \mathrm{MV} / \mathrm{m}$. Selected emitters localized in FESM and identified in SEM have been categorized depending on their shape and impurity content. The $I-V$ curves of most emitters confirm the modified $\mathrm{FN}$ theory with reasonable $\beta$ and $S$ values. In comparison, particulates showed more current instabilities than surface protrusions, as expected from their relative mechanical stability. $E_{\text {on }}$ values of all emitters have been significantly improved by DIC. The highest values were achieved for CryNb, i.e., 88-167 MV/m before and 116-186 MV/m after DIC, thus underlining the benefit of mirrorlike surfaces for reduced FE. Large-grain $\mathrm{Nb}$ samples do not show any emission from grain boundaries up to $250 \mathrm{MV} / \mathrm{m}$. SEM images of typical emitters prove that DIC removes particulates down to $400 \mathrm{~nm}$ size and partially smoothens the edges of protrusions.

\section{ACKNOWLEDGMENTS}

We would like to acknowledge A. Aspart and C. Antoine from CEA Saclay for electropolishing of the polycrystalline $\mathrm{Nb}$ samples, and A. Brinkmann and J. Ziegler from DESY as well as R. Grimme and C. Zorne from Fraunhofer IPA for experimental support of the DIC apparatus. We are thankful to the Electrical Engineering Department at BUW for access to SEM and EDX facilities. Stimulating discussions with W. Singer and D. Proch from DESY as well as C. S. Pandey from BUW are gratefully acknowledged. The support of the
European Community Research Infrastructure Activity under FP6 "Structuring the European Research Area" program (CARE, Contract Number RII3-CT-2003-506395) is gratefully acknowledged.

${ }^{1}$ R. V. Latham, High Voltage Vacuum Insulation: Basic Concepts and Technological Practice (Academic, London, 1995).

${ }^{2}$ H. Padamsee, J. Knobloch, and T. Hays, RF Superconductivity for Accelerators (Wiley, New York, 1998).

${ }^{3}$ R. J. Noer, Appl. Phys. A 28, 1 (1982).

${ }^{4}$ R. H. Fowler and L. Nordheim, Proc. R. Soc. London, Ser. A 119, 173 (1928).

${ }^{5}$ R. J. Noer, Ph. Niedermann, N. Sankarraman, and O. Fischer, J. Appl. Phys. 59, 3851 (1986).

${ }^{6}$ E. Mahner, N. Minatti, H. Piel, and N. Pupeter, Appl. Surf. Sci. 67, 23 (1993).

${ }^{7}$ N. Pupeter, T. Habermann, A. Kirschner, E. Mahner, G. Müller, and H. Piel, Appl. Surf. Sci. 94-95, 94 (1996).

${ }^{8}$ N. Pupeter, A. Göhl, T. Habermann, A. Kirschner, E. Mahner, G. Müller, and H. Piel, Part. Accel. 53, 77 (1996).

${ }^{9}$ T. Wang, C. E. Reece, and R. M. Sundelin, J. Vac. Sci. Technol. B 21, 1230 (2003).

${ }^{10}$ A. Dangwal, D. Reschke, and G. Müller, Physica C 441, 83 (2006).

${ }^{11}$ R. A. Bowling, in Particles on Surfaces, edited by K. L. Mittal (Plenum, New York, 1988), Vol. 1, p. 129.

${ }^{12} \mathrm{P}$. Kneisel and B. Lewis, in Proceedings of the 7th Workshop on RF Superconductivity, Gif sur Yvette, France, edited by B. Bonin (1995), p. 311.

${ }^{13}$ R. Sherman and W. Whitlock, J. Vac. Sci. Technol. B 8, 563 (1990).

${ }^{14}$ R. Sherman, J. Grob, and W. Whitlock, J. Vac. Sci. Technol. B 9, 1970 (1991).

${ }^{15}$ R. Sherman, D. Hirt, and R. Vane, J. Vac. Sci. Technol. A 12, 1876 (1994).

${ }^{16}$ D. Proch, D. Reschke, B. Guenther, G. Müller, and D. Werner, in Proceedings of the 10th Workshop on RF Superconductivity, Tsukuba (2001), p. 463.

${ }^{17}$ L. Layden and D. Wadlow, J. Vac. Sci. Technol. A 8, 3881 (1990).

${ }^{18}$ C. Suzuki, T. Nakanishi, S. Okumi, T. Gotou, K. Togawa, F. Furuta, K. Wada, T. Nishitani, M. Yamamoto, J. Watanabe, S. Kurahashi, K. Asano, H. Matsumoto, M. Yoshioka, and H. Kobayakawa, Nucl. Instrum. Methods Phys. Res. A 462, 337 (2001).

${ }^{19} \mathrm{D}$. Reschke, in Proceedings of the 12th Workshop on RF Superconductivity, Cornell University, SUP03 (2005).

${ }^{20}$ D. Lysenkov and G. Müller, Int. J. Nanotechnol. 2, 239 (2005).

${ }^{21}$ T. Habermann, Ph.D. Thesis, University of Wuppertal (1999).

${ }^{22}$ D. Werner and C. Zorn, in Proceedings of Precision Cleaning, Clean Tech, Frankfurt (2000).

${ }^{23}$ D. Werner, P. Fode, and H. Schöne, in "Cleanroom Technology," issue Feb. 2001, p. 35.

${ }^{24}$ A. Brinkmann, J. Iversen, D. Reschke, and J. Ziegler, in Proceedings of the European Particle Accelerator Conference, Edinburgh, 2006), p. xx.

${ }^{25}$ T. Habermann, A. Göhl, D. Nau, G. Müller, H. Piel, and M. Wedel, Part. Accel. 61, 137 (1998). 